\title{
CORRECTION
}

\section{COEXISTENCE IN THRESHOLD VOTER MODELS}

\author{
By Thomas M. LiggetT
}

Annals of Probability (1994) 22 764-802

The theorem proved in that paper asserts that every threshold voter model on $Z^{d}$ coexists, except for the one that is nearest neighbor in one dimension (which is known to cluster). The proof given there divides into two main parts:

(a) Show that there is a probability density $f$ on the positive integers that satisfies a particular convolution equation [(3.6) in the paper], and that this density and the renewal sequence have certain monotonicity and convexity properties, and

(b) Use part (a) to show that the renewal measure on $\{0,1\}^{Z}$ corresponding to $f$ satisfies a collection of inequalities: $Q(A) \geq 0$ for all finite $A \subset Z$, where $Q$ is defined in (3.4).

Part (a) of the proof given in the paper was computer assisted, but correct. Part (b) was analytic, but incorrect.

The error in part (b) of the proof occurred in attempting to show that the bilinear expression (3.24) is nonnegative for all choices of $L_{A}$ and $R_{A}$ for which the corresponding $L$ and $R$ satisfy the inequalities (3.25). The inequalities are linear, so in checking such a statement, it is enough to check it at the extreme points of the convex sets determined by them. In the proof, however, in order to reduce the dimensions of the convex sets involved, some simplifications were made that rendered the expression that was originally bilinear, no longer bilinear. Therefore, for the simplified expression, it is no longer enough to check the inequalities at the extreme points, as was done in the paper. In fact, these inequalities are not always correct.

An example that satisfies (3.25), but for which (3.24) is negative, is

$$
\begin{aligned}
L(m-2) & =L(m-1)=R(m+2)=R(m+1)=26 \\
L(m) & =R(m)=27 \\
L(m+1) & =R(m-1)=28 \\
L(m+2) & =R(m-2)=29
\end{aligned}
$$

Received February 1999. 
and correspondingly,

$$
\begin{aligned}
L_{A}(m-2) & =L_{A}(m-1)=R_{A}(m+2)=R_{A}(m+1)=26 \\
L_{A}(m) & =R_{A}(m)=14 \\
L_{A}(m+1) & =R_{A}(m-1)=15 \\
L_{A}(m+2) & =R_{A}(m-2)=\frac{21}{4}+26 \beta .
\end{aligned}
$$

The author discovered the error in writing his new book, which is listed in the References below. A corrected proof is included in Part II of the book, but is too involved to reproduce here. The treatment in the book is also improved in that part (a) of the proof is entirely analytic and no longer relies on a computer. The section dealing with this problem is posted on the author's web page at http://www. math.ucla.edu/ tml / .

\section{REFERENCE}

Liggett, T. M. (1999). Stochastic Interacting Systems: Contact, Voter and Exclusion Processes. Springer, Berlin.

Department of Mathematics UNIVERSITY OF CALIFORNIA Los ANGeles, CALIFornia 90095 E-MAIL:tml@math.ucla.edu 